\title{
Análisis discursivo en sistemas híbridos de medios: una aproximación metodológica
}

\author{
Discourse analysis in the hybrid media system: a methodological approach
}

Recibido: Marzo 2020 Aceptado: Agosto 2020 Publicado: Diciembre 2020

\author{
Daniela Ibarra Herrera \\ Pontificia Universidad Católica de Chile, Chile \\ dvibarra@uc.cl
}

\begin{abstract}
Resumen: Este trabajo propone una aproximación metodológica para el estudio de discursos en el sistema híbrido de medios (Chadwick, 2013). El sistema híbrido de medios se define como un nuevo tipo de sistema en el que los medios tradicionales y emergentes se ensamblan, cambian y fluyen gradualmente para crear nuevas formas. Una forma de hibridación es la relación entre redes sociales y televisión, en la que ambos medios se modelan y construyen mutuamente. Diversos estudios (Gruber, 2017; Vaccari et al., 2016) han demostrado la importancia del estudio del sistema híbrido de medios para explorar la construcción de significados en los nuevos contextos de medios masivos, especialmente en relación a temas políticos. Este estudio se enmarca en un paradigma cualitativo que incorpora los principios de los estudios críticos del discurso para el análisis de recursos semióticos utilizados en 30.000 tweets marcados con hashtags de cinco programas televisivos políticos chilenos emitidos durante el 2016, que promueven el debate y la participación del público online. A partir del análisis de los textos online y los programas de televisión se pudieron establecer que las estrategias argumentativas y recursos utilizados constituyen una expansión del debate político televisado y un espacio de interacción de los usuarios de redes sociales con los medios tradicionales de comunicación. La decisión de involucrar directamente a estos usuarios en programas de televisión tiene implicancias para la comprensión de los límites simbólicos de la política. La inclusión de nuevos actores tiene el potencial de modelar la calidad del debate y la argumentación en la esfera pública digitalmente mediada.
\end{abstract}

Palabras clave: Estudios del discurso digital - Sistema híbrido de medios - Dual screening - Estudios críticos del discurso en redes sociales

Citación: Ibarra Herrera, D. (2020). Análisis discursivo en sistemas híbridos de medios: una aproximación metodológica. Logos: Revista de Lingüística, Filosofía y Literatura, 30(2), 314-330. doi. org/10.15443/RL3024

Dirección Postal: Campus San Joaquín, Av. Vicuña Mackenna 4860, Macul, Santiago, Chile 


\begin{abstract}
This paper proposes a methodological approach to the study of discourses in the hybrid media system (Chadwick, 2013). The hybrid media system is defined as a new type of system in which traditional and emerging media are assembled, changed and flow gradually to create new forms. One form of hybridization is the relationship between social networks and television, in which both media model and build each other. Various studies (Gruber, 2017; Vaccari et al., 2016) have demonstrated the importance of the study of hybrid media system to explore the construction of meanings in new media contexts, especially in political contexts. This study is part of a qualitative paradigm that incorporates the principles of critical discourse analysis for the analysis of semiotic resources used in 30,000 tweets with hashtags of five Chilean political television programs broadcast during 2016, which promote online public debate and participation. Based on the analysis of online texts and television programs, it was established that the argumentative strategies and resources used constituted an expansion of the televised political debate and a space for interaction of social network users with traditional media. The decision to directly involve these users in television programs has implications for the understanding of the symbolic limits of the policy. The inclusion of new actors has the potential to model the quality of debate and argumentation in the digitally mediated public sphere.
\end{abstract}

Keywords: Digital discourse analysis - Hybrid media system - Dual screening Social Media Critical Discourse Studies

\title{
1. Introducción
}

El presente artículo propone una aproximación metodológica para el estudio de discursos en el sisitema híbrido de medios, en el cual los medios nuevos y tradicionales se conciben como un continuo en el que se combinan, coevolucionan y crean nuevas formas (Chadwick, 2013). Una de las prácticas sociales asociadas a esta nueva concepción de las relaciones mediales es el llamado dual-screening. Esta práctica se define, a grandes rasgos, como el uso de las redes sociales para acceder a información, socializar o comentar programas de televisión (Gil de Zúñiga et al., 2015). Para explorar el fenómeno del dual-screening se estudiarán 39,684 tweets que utilizan los hashtags oficiales promovidos por cinco programas de televisión políticos chilenos, correspondientes a tres meses del año 2016. Esta propuesta metodológica analiza los datos cualitativamnete en dos etapas, la primera, en la cual se identificaron los principales temas y características formales del conjunto total de datos, y una segunda etapa en la cual se analizó una muestra de 140 tweets en donde se exploraron las principales estrategias argumentativas de los textos.

\section{Una aproximación a los sistemas híbridos de medios}

El auge de las redes sociales ha influido no solo en el desarrollo de nuevas formas de comunicarse, sino también en la evolución de los medios de comunicación tradicionales (Chadwick, 2013). Una de las principales diferencias entre los medios de comunicación masivos tradicionales y las nuevas formas de comunicación, como redes sociales y plataformas online, es su funcionamiento y la relación que logran establecer entre los distintos participantes. Los medios de comunicación masiva más tradicionales tienden a funcionar como un mecanismo de transmisión de uno a 
muchos (como periódicos, libros, televisión, películas, radio, etc.), a diferencia de las plataformas de redes sociales, que pueden entregar contenidos en una red de participantes donde cualquiera puede participar, publicar y que, potencialmente, puede llegar a grandes audiencias (Page et al., 2014; Castells, 2009). Los medios de comunicación tradicionales o de masas, por otro lado, se han desarrollado para difundirse ampliamente en la sociedad de forma unidireccional. Aunque los medios tradicionales tienen mecanismos para comunicarse con sus públicos, centrados en marketing, ventas o servicios al cliente como cartas, correos electrónicos y llamadas telefónicas, Chadwick (2013) argumenta que este tipo de medios no se han concebido como un espacio comunicativo interpersonal. Como plantea Larsson (2013), el periodismo de los medios masivos de comunicación y la forma en la que se entrega la información siempre han sido determinados por la tecnología y cambian constantemente con el surgimiento de nuevas prácticas sociales. Hoy en día existen diferentes prácticas para que las personas accedan a la información e interactúen con otros medios, incluso los más tradicionales. Castells (2009) explica que hay un proceso de convergencia de diversos modos en el que las líneas entre los diferentes medios se tornan cada vez más borrosas. Siguiendo esto, los principales medios de comunicación han incorporado elementos de redes sociales para mejorar la experiencia de la audiencia. Con el surgimiento de estas nuevas prácticas de medios, es posible hablar sobre una nueva era de comunicación con medios híbridos en lugar de géneros y modos completamente establecidos o aislados.

Dentro de este contexto, surge la noción del sistema híbrido de medios (Chadwick, 2013), que propone una comprensión conceptual de las relaciones de poder entre los medios más recientes y los clasificados como medios más tradicionales. Chadwick (2013) propone que estas relaciones entre las lógicas de los diversos medios, como géneros, modos, plataformas, entre otros, vinculan los campos de los medios y la política. El concepto de hibridación aplicado en los medios y contextos políticos puede ofrecer complejidad y, en cierta medida, explica los nuevos paradigmas en estos campos. Este planteamiento propone rechazar las dicotomías tradicionales y analizar los fenómenos vinculados a la política y los medios como un sistema en constante transición, evolución y reconstrucción.

Aunque los conceptos de medios nuevos y antiguos son relativos, la integración entre los diferentes tipos de medios no solo está relacionada con las plataformas digitales. El proceso de integración de las diferentes lógicas de los medios promueve la coevolución de diversas prácticas en lugar de la sustitución de los nuevos medios por sobre los antiguos. Una lógica de medios puede definirse como una forma de comunicación y el proceso a través del cual los medios transmiten y comunican información (Altheide, 2013). Un ejemplo de este tipo de coevolución son las nuevas posibilidades $y$, a veces, la fusión de funciones, como se encuentran en los smartphones o tabletas. El sistema de medios híbridos, como explica Chadwick (2013), involucra diferentes prácticas que se encuentran en recreación continua, reconstrucción y remodelación, creando un entorno de medios policéntrico y fragmentado.

El concepto del sistema híbrido de medios crea un marco conceptual que explica la dinámica entre los diferentes medios y cómo la integración de los medios nuevos y antiguos está creando nuevas relaciones de poder, nuevas formas de construir significados y dar forma al quehacer político. El sistema híbrido de medios, como lo propone Chadwick (2013), ha sido ampliamente estudiado en contextos de habla inglesa, específicamente en los Estados Unidos y el Reino Unido. El autor afirma que el estudio de esos contextos es importante por su papel en la historia de las democracias liberales, pero poco se ha explorado en democracias recientes o países del sur global. Es por esto que toma relevancia la exploración del concepto del sistema híbrido de medios en un contexto latinoamericano, específicamente en el chileno.

Chadwick (2013) afirma que el poder, como un concepto esencial para comprender la política, es ejercido por aquellos que utilizan con éxito la transformación e interdependencia de las lógicas de medios nuevos y antiguos y gestionan las formas de información para satisfacer sus objetivos. Desde esta perspectiva, los medios ayudan a dar forma a la política y permiten la participación de los ciudadanos en la esfera política. Con la convergencia y las interacciones en estos nuevos tipos de medios, los ciudadanos pueden interactuar y participar en el debate político. 


\subsection{Dual-screening como práctica social}

Dentro de la evolución de los diversos géneros y el sistema híbrido de medios, surge la idea de el dual-screening como una forma híbrida de comunicación que puede vincular diferentes medios, nuevos y antiguos (Vaccari, Chadwick \& O'Loughlin, 2015). Este fenómeno ha sido descrito por como el uso de un dispositivo digital como un computador, smartphone o tableta para acceder a Internet, específicamente a redes sociales, mientras se ve televisión con el objetivo de participar, obtener información o discutir sobre lo que están viendo, por ejemplo, los tweets generados con relación a diversos programas de televisión. Esta tendencia es cada vez más común e incluso es promovida por los diferentes programas de televisión, en los que los productores y las emisoras incitan al público a usar las redes sociales para comentar y discutir el programa en sí (Giglietto \& Selva, 2015). El nivel de importancia que hoy se otorga a las redes sociales es tal, que el éxito de un programa se puede medir a través de su presencia en las redes sociales, como el número de menciones o, si se convierte en Trending Topic (Marchetti \& Ceccobelli, 2015; Cameron \& Geidner, 2014). Para Vaccari, Chadwick y O'Loughlin (2015), el dual-screening se define como un "conjunto complejo de prácticas que involucran la integración y el intercambio entre medios de comunicación en vivo, como programas de televisión, y redes sociales" (1041) y es el resultado de una rápida masificación de las plataformas de redes sociales y los intentos de renovación y adaptación de la televisión como medio.

Un ejemplo de esta práctica son las interacciones en redes sociales asociadas a programas de televisión, como los tweets analizados en esta investigación, los cuales están marcados con los hashtags de cinco programas de televisión chilenos que promueven la participación de la audiencia en línea, mencionando los hashtags en pantalla. El estudio de estos textos producidos en el contexto de hibridación medial, más específicamente en la práctica de dual-screening, pueden contribuir a la exploración de nuevas formas de comunicar en este nuevo sistema y cómo se genera la discusión en relación con diversos temas.

\subsection{Hacia una metodología de estudio del dual-screening}

Para explorar cómo los usuarios de redes sociales interactúan y discuten con relación a programas de televisión es necesario recolectar textos generados en este contexto para su análisis. Este fenómeno ha sido analizado principalmente desde los estudios de medios y comunicación, sin tener en cuenta, muchas veces los diversos elementos lingüísticos involucrados en esta práctica social. En relación a los propósitos de esta investigación y con el fin de explorar los diversos recursos utilizados por los usuarios de Twitter para construir sus textos, se optó por una recolección manual de datos desde la red social.

Aunque la recolección manual desde Twitter puede considerarse limitada en comparación con la extracción automatizada de software, este método ofrece otras posibilidades para los investigadores, como recuperar datos de fechas específicas o incluir diferentes modalidades en los textos. Como sostienen Latzko-Toth, Bonneau y Millette (2017), una ventaja de la recopilación manual de datos es que permite a los investigadores familiarizarse con los datos en el formato "nativo", esto quiere decir, como se ve para los usuarios de la plataforma. Además, este tipo de recolección permite la exploración de diferentes tipos de contenidos y elementos, como vínculos, imágenes, colores, entre otros.

La mayoría del software para extraer datos de las redes sociales exporta los tweets en texto sin formato (principalmente txt.) para el análisis, aunque esto resulta muy útil, en el caso de esta investigación, se optó por la recopilación manual. Como se indicó anteriormente, uno de los objetivos de esta investigación es explorar diferentes recursos semióticos, por esa razón, la recopilación manual en formato "nativo" brinda la oportunidad de abordar los datos con la mayoría de los elementos que están presentes para el usuario, incluidos diferentes recursos y modos semióticos.

Para la primera etapa de análisis se categorizaron las principales características formales y 
los temas presentes en 39,684 tweets. Después del proceso de codificación, para la segunda etapa, se analizó en detalle una muestra de 140 tweets seleccionados al azar de la recopilación de datos total, seleccionando aleatoriamente 10 por cada programa de televisión en cada mes. Este proceso ayudó a reducir el tamaño de los datos para explorar con más detalle los tweets relacionados con la práctica de dual screening en la segunda etapa de la investigación.

Como plantean Barton y Lee (2013) los textos presentes en Twitter, o tweets, se han definido como un tipo de microblog que posee una extensión limitada. Estas plataformas permiten a los usuarios publicar mensajes cortos, de no más de 140 caracteres en el momento de la recopilación de datos (ahora permite 280). Los usuarios también pueden suscribirse a las cuentas de otros usuarios y recibir sus actualizaciones en una "línea temporal" (timeline). La naturaleza limitada del microblog ha hecho que este tipo de comunicación sea de especial interés para los investigadores ya que los usuarios necesitan aprovechar al máximo sus recursos para entregar mensajes efectivos (Zappavigna, 2012).

\subsection{La "primera pantalla": descripción y caracterización de los programas de televisión}

Esta investigación se centra en los datos de las redes sociales generados en relación con los programas de televisión políticos en Chile, por lo que es importante caracterizar los programas de televisión que promueven y alientan la participación de las redes sociales mediante la promoción de hashtags en pantalla y la inclusión de tweets en los programas de televisión. Las publicaciones de Twitter generados por los espectadores están estrechamente relacionadas con la discusión llevada a cabo en los programas de televisión, en ese sentido, esto proporciona un contexto para la discusión en línea. La información de la estructura y las características principales del programa de televisión pueden ser útiles para proporcionar una mejor imagen de los datos (Roberts, 2008). Como boyd y Crawford (2012) mencionan, las prácticas sociales en las redes sociales no se pueden entender de manera aislada de las diversas perspectivas y actores sociales que participan creando significados. Por esa razón, para entender los discursos políticos generados por los espectadores es necesario incluir una descripción de los programas de televisión relacionados con los datos.

\section{Procedimientos de análisis}

La primera etapa del análisis consiste en la identificación de los tópicos y las características formales en todo el conjunto de datos para tener una visión general de la práctica de dual screening en el contexto de la política chilena. El examen de las características y temas formales contribuye a la comprensión de cómo las personas usan Twitter para participar en el debate político en el contexto de los medios híbridos y qué recursos semióticos están involucrados en estas prácticas sociales.

Para la segunda etapa de esta investigación, se analizó en detalle una muestra aleatoria de los datos de las redes sociales codificados en la primera etapa. Los tweets se analizaron utilizando el análisis del discurso de la historia (Reisigl \& Wodak, 2016) para explorar la interacción y las estrategias argumentativas llevadas a cabo en este tipo de datos sobre la política chilena. La Tabal 1 muestra el tipo de análisis que se llevará a cabo para cada conjunto de datos.

Este estudio comprende un primer análisis de los tweets que involucra el procesamiento de grandes cantidades de datos. Un enfoque de la lingüística de corpus podría ser muy útil para procesar grandes corpora (véase, por ejemplo, Zappavigna, 2017; Baker, 2006). Además, podría ayudar a triangular los datos de un análisis crítico del discurso y ampliar la representatividad del estudio (Baker et al., 2008). El uso de la lingüística de corpus para explorar datos de redes sociales ha contribuido al estudio de diferentes prácticas sociales en línea, especialmente en Twitter (por ejemplo, Potts et al., 2014; Hardaker \& McGlashan, 2016; Page, 2012; Zappavigna, 2012). Sin embargo, este tipo de análisis puede pasar por alto algunas características que pueden ser útiles para el análisis de la argumentación, por ejemplo, la argumentación no verbal, como los recursos multimodales presentes en los tweets (imágenes, emojis, entre otros). Por 
esta razón, y para complementar el creciente cuerpo de investigación basada en corpus, empleo una perspectiva cualitativa para estudiar la argumentación en estos contextos.

Tabla 1. Conjunto de datos y procedimientos

\begin{tabular}{|c|c|c|}
\hline Tipo de datos & Análisis & Cantidad de datos \\
\hline $\begin{array}{l}\text { Datos extraídos de Twitter } \\
\text { Tres meses de tweets marcados } \\
\text { con los hashtags de cada } \\
\text { programa de TV. } \\
\text { Estado Nacional, Ciudadanos, } \\
\text { Entrevista Verdadera, Mejor } \\
\text { Hablar de Ciertas Cosas y El } \\
\text { Informante }\end{array}$ & $\begin{array}{l}\text { Codificación de tópicos y } \\
\text { características formales }\end{array}$ & $\begin{array}{l}\text { 39,684 tweets ( } 5335 \text { páginas de } \\
\text { PDF) }\end{array}$ \\
\hline $\begin{array}{l}\text { Muestra de tweets } \\
\text { Seleccionados aleatoriamente } \\
\text { del conjunto de datos }\end{array}$ & $\begin{array}{l}\text { Análisis detallado de las } \\
\text { estrategias argumentativas }\end{array}$ & $\begin{array}{l}140 \\
\text { (10 tweets por cada programa } \\
\text { de televisión en los meses } \\
\text { seleccionados) }\end{array}$ \\
\hline
\end{tabular}

\subsection{Proceso de codificación}

La primera etapa de análisis en la investigación se caracteriza por la codificación de toda la recolección de datos. En la investigación cualitativa, un código se define como una palabra o frase corta que asigna un atributo simbólicamente a una parte de los datos, generalmente, visual o lingüísticos (Saldaña, 2013). Los códigos son construcciones generadas por los investigadores con el objetivo de capturar el contenido primario y el significado de los datos y, por lo tanto, crear patrones, categorías y otros procesos analíticos. El proceso de codificación establece un "vínculo crítico" entre los datos y su posible explicación de significado. Para Flick (2008), el proceso de codificación consiste en porciones de datos de la etiqueta y crea grupos con otros datos. Este proceso puede definirse como un acto interpretativo, y por esta razón, la codificación para crear un sistema de clasificación no es una ciencia exacta. Los códigos están diseñados para crear categorías o familias con algunas características compartidas.

\subsection{Codificación en ATLAS.ti}

En la primera etapa del análisis de esta investigación, se analizaron los tweets relacionados con los hashtags de los programas de televisión del comienzo, la mitad y el final de la temporada de televisión chilena en 2016. Se procesaron los datos de las redes sociales extraídos del sitio web. con la ayuda del software ATLAS.ti, un software de análisis de datos cualitativos asistidos por computadora o CADQAS. El proceso de codificación consiste en la clasificación de los datos en diferentes categorías que se pueden analizar y agrupar en categorías más amplias. A pesar de que el proceso de codificación y la categorización son realizados manualmente por los investigadores, este tipo de software facilita el análisis sistemático y la recuperación de la información.

Esta clasificación ayuda a reconocer los intereses más relevantes de los programas de televisión políticos tanto como sus audiencias. Como propone Silverman (2016), el análisis de contenido de los datos puede ayudar a abordar diferentes fenómenos de manera sistemática. El análisis de este tipo de discurso requiere estudiar la combinación e integración de la forma y el contenido para comprender, explicar y deconstruir los mensajes en contextos políticos. La codificación en este contexto proporciona un enfoque conceptual de los datos para describirlos y comprender los diferentes elementos del fenómeno de dual screening como una práctica social.

\subsection{Estudio piloto}

El estudio piloto permite a los investigadores explorar los datos y probar el proceso de 
codificación, antes de la codificación de toda la recopilación de datos. Como resultado del estudio piloto, se crearon 43 códigos para identificar temas y características formales; 30 relacionadas con características formales en los tweets y 13 con temas presentes en el texto. En los tweets analizados, hay diferentes características formales que pueden describirse como recursos semióticos que ayudan a los productores a construir sus textos en el debate online.

Con la información obtenida del estudio piloto, se pudo refinar y volver a elaborar los códigos utilizados. El objetivo de este procedimiento era hacer que el proceso de codificación de todo el conjunto de datos fuera más eficiente, coherente y sistemático. Después de la primera etapa de codificación, como propone Saldaña (2013), el proceso de codificación debe probarse y perfeccionarse en el segundo ciclo de codificación.

\subsection{Codificación temática de los textos}

La identificación de temas, mediante el análisis de contenido y la aplicación de un conjunto sistemático de códigos en la recolección de datos, permite una aproximación general a las prácticas en redes sociales relacionadas con programas de televisión políticos. Los códigos emergentes ayudan a ilustrar las principales características presentes en las conversaciones en línea y los temas más relevantes para los usuarios de Twitter en relación con los debates televisados.

Los temas presentes en los textos fueron organizados a partir de la codificación de 39,684 tweets se clasificaron en dos categorías, procesos sociales y actores sociales. Estas categorías si bien se inspiran en los trabajos de van Leeuwen (2008), se basan exclusivamente en los temas emergentes de los datos. Los códigos agrupados emergentes de la codificación de los textos estudiados se presentan en laa Figura 1 y Figura 2.

Figura 1. Procesos sociales identificados en los textos

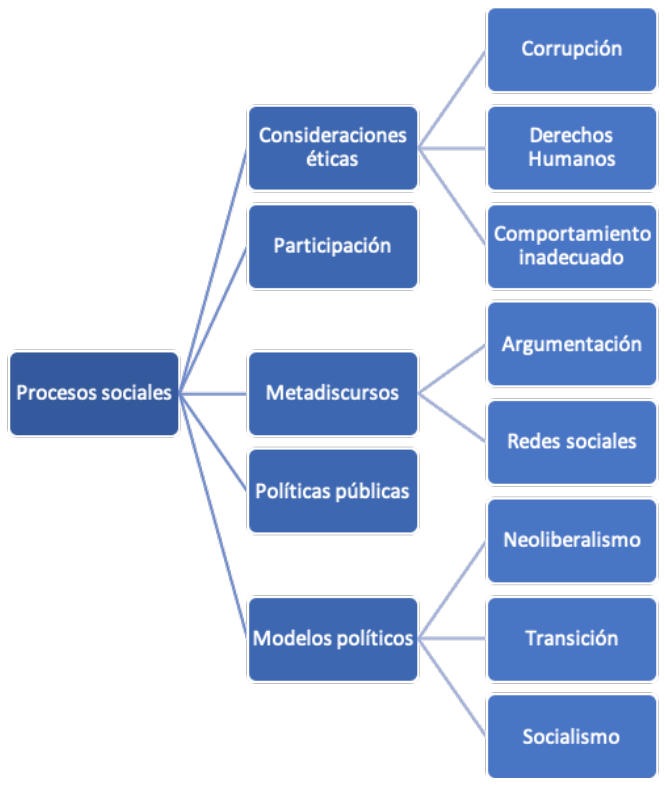

Las figuras 1 y 2 muestran la clasificación de los códigos temáticos emergentes en el proceso de codificación. Estos se agruparon actores y procesos sociales, estas categorías permiten tener una mirada general de los temas presentes en los textos producidos en este contexto de hibridación medial. Las frecuencias de estos códigos se presentan en la Figura 3. 
Figura 2. Actores sociales identificados en los textos

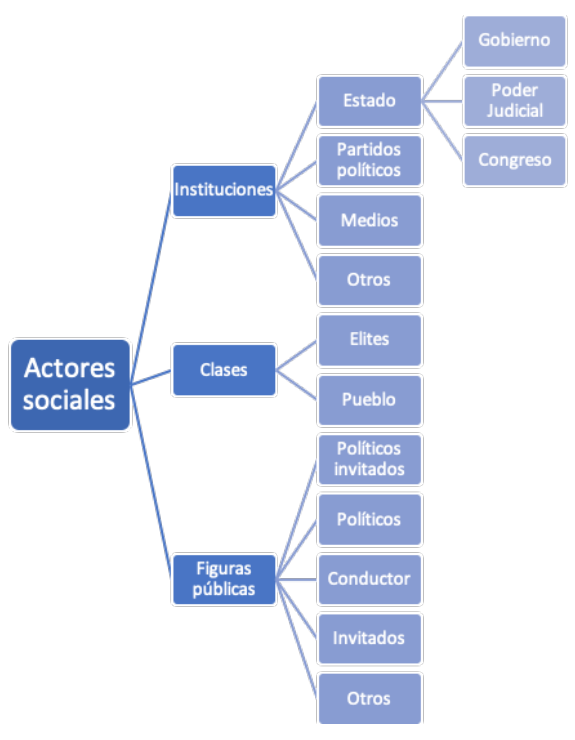

Figure 3. Frecuencia temática en los tweets analizados

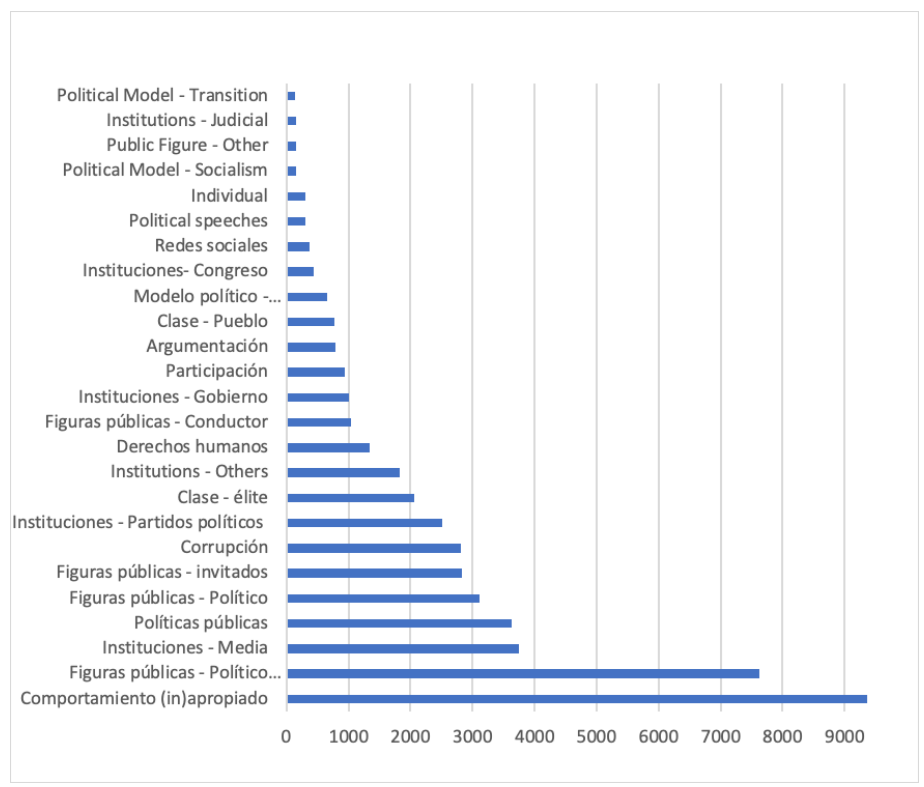

La figura 3 muestra la frecuencia de tweets por código que indican temas en los datos. Los temas más frecuentes en las discusiones mediadas digitalmente fueron los códigos relacionados con preocupaciones éticas, a saber, comportamiento (in)apropiado y corrupción, además de los ligados a figuras públicas que participan en los progrmas. Estos códigos se refieren a casos particulares, pero también al comportamiento general de la clase política, figuras públicas e instituciones, y generalmente se utilizan para criticar a los que están en el poder. Los hashtags de los programas pueden usarse en estos casos para interactuar con una comunidad 
de espectadores que se preocupa por la esfera política y, por lo tanto, llegar a audiencias más amplias para los propios objetivos políticos de los usuarios.

\section{Rasgos formales como recursos semióticos}

Los recursos semióticos son parte de un sistema que evoluciona para servir a diferentes funciones sociales. Según Lemke (2009), el estudio de la semiótica y cómo los recursos semióticos construyen significados es necesario para entender cómo funciona el poder político. Este tipo de análisis puede ayudar a explicar cómo se ejerce y distribuye el poder. Estos recursos se pueden apreciar en la Figura 4.

Figura 4. Características formales de los textos analizados

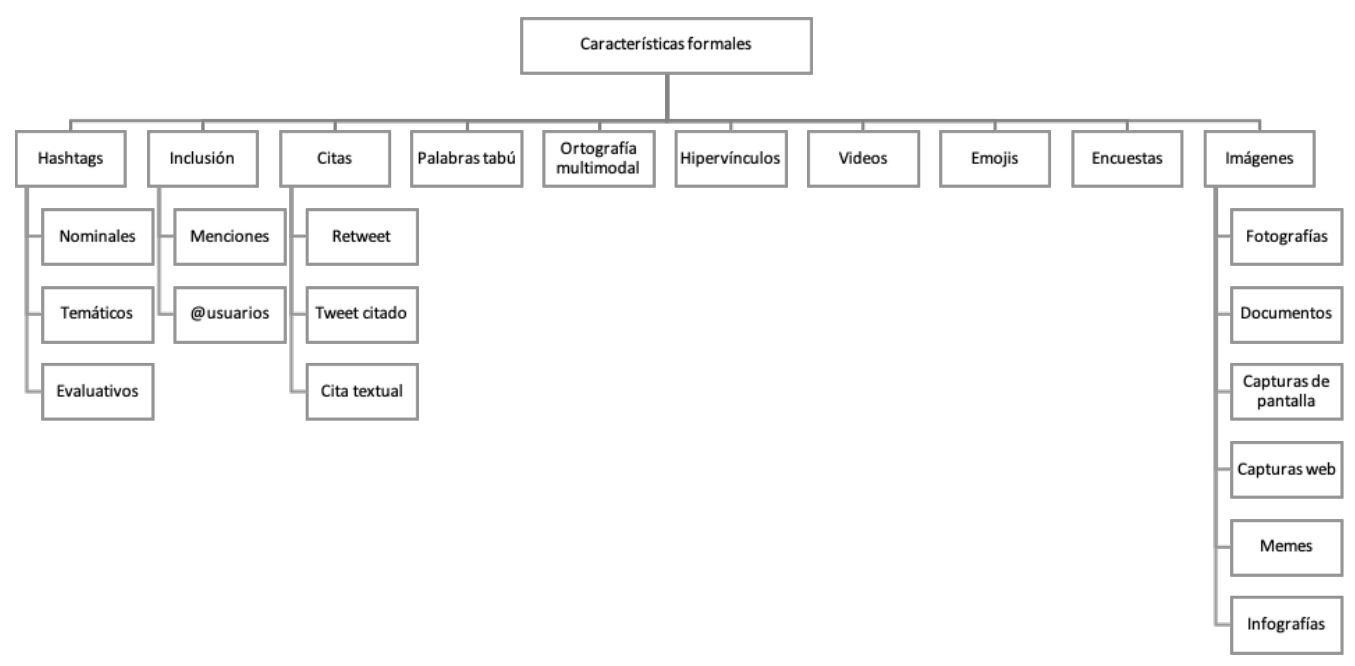

Como se observa en la figura 4, los usuarios de Twitter pueden recurrir a diferentes tipos de recursos para construir sus argumentos, estos recursos fueron clasificados como verbales, visuales e hipertextuales. Dentro de los recursos visuales identificados en los tweets se encuentran los emojis, diferentes tipos de imágenes como fotografías, caricaturas, memes, capturas de pantalla de los programas, de otros sitios web e infografías. Los recursos verbales por su parte, se identifican principalmente como recursos para incluir otros y la voz de otros en los tweets. entre estos se encuentran las menciones e inclusion de usuarios de la red de Invitados, conductores de los programas, figuras públicas, partidos políticos o instituciones públicas; insercion de citas en los tweets, usualmente de los invitados del programa u otras figuras públicas y tweets citados, también conocido como retweeting.

Siguiendo a Lemke (2009), los recursos hipertextuales se pueden definir como un tipo de texto mediado digitalmente que vincula una unidad con otra y establece diferentes relaciones entre los modos. Estas relaciones multimodales no pueden considerarse como una yuxtaposición de texto y otros recursos, sino más bien como un nuevo tipo de modalidad. Estos recursos permiten a los usuarios interactuar con la plataforma y saltar (o, según Lemke, recorrer) a otros textos, imágenes, sitios web o plataformas, que en este caso fueron identificados como hyperlinks y hashtags: temáticos, nominativos y evaluativos.

Si bien los diversos recursos semióticos identificados en los datos se clasificaron en verbales, visuales e hipertextuales, estas categorías no son del todo estáticas y en ciertos casos se superponen como se aprecia en la figura. Por ejemplo, un hashtag puede vincular la conversación con otros textos, pero a la vez incluir al sujeto del tweet, reemplazando el nombre. Es por 
esto que la clasificación anterior sirve como guía para la exploración de los diversos recursos semióticos analizados en este contexto, pero no se constituye como una taxonomía estática, ya que tiene en cuenta las diversas funciones que pueden desempeñar los diversos recursos en este tipo de textos.

Figura 5. Clasificación de recursos semióticos

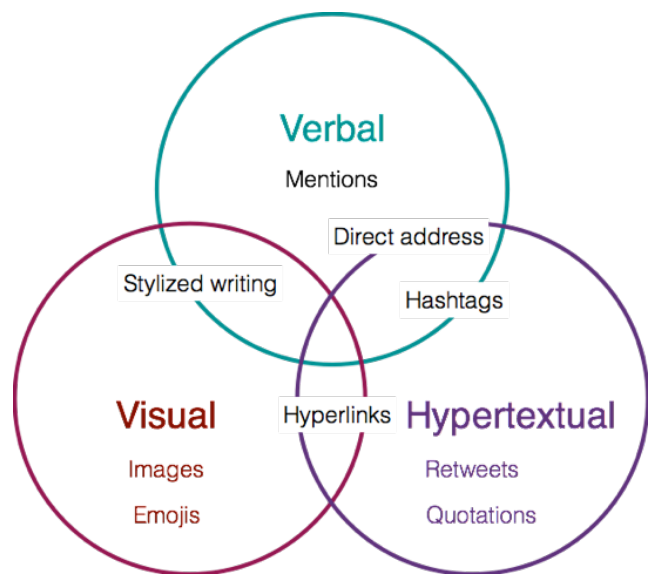

\section{Segunda etapa de análisis: Argumentación y estrategias de discurso en el contexto de dual screening.}

En la segunda etapa de esta investigación, se realizó un análisis detallado de una muestra de tweets relacionados con los programas políticos chilenos. Como afirma Marwick (2014), el análisis del discurso en medios digitales, y específicamente su relación con el poder y la ideología, debe llevarse a cabo en una muestra relativamente pequeña, que permita a los investigadores explorar los textos con más detalle y profundidad. Por esta razón, la segunda etapa del análisis comprendió una pequeña muestra de la recopilación de datos más amplia, analizada en la primera etapa. Este análisis se centró en las estrategias de argumentación presentes en los diferentes textos y su relación con la discusión en la televisión. Para seleccionar los tweets para el análisis detallado, se utilizó una fórmula aleatoria para recopilar una muestra de 140 tweets extraídos del gran conjunto de datos, diez de cada programa de televisión analizado por mes. Unger, Wodak y KhosraviNik (2016) argumentan que, debido a la gran cantidad de datos generados para los sitios de redes sociales, uno de los pasos metodológicos del enfoque histórico-discursivo para estudiar el discurso es la reducción de tamaño y la selección de los datos.

\subsection{Enfoque discursivo-histórico}

Como uno de los principales objetivos de esta investigación es explorar y comprender las prácticas de argumentación en contextos digitalmente mediados, el análisis de las estrategias argumentativas y los recursos involucrados en los tweets sobre política en las redes sociales relacionadas con programas de televisión es un elemento clave en esta investigación. Para lograr esto, el enfoque histórico-discursivo (en adelante DHA) (Reisigl \& Wodak, 2016) puede ser una forma útil de obtener una comprensión más holística del fenómeno a través del análisis de diferentes estrategias discursivas y especialmente argumentativas. Hay estudios (por ejemplo, KhosraviNik \& Unger, 2016; KhosraviNik, 2017) que muestran las posibles aplicaciones del enfoque discursivo-histórico a la comunicación en las redes sociales, específicamente relacionado con la política.

Para integrar los antecedentes históricos del análisis y explorar cómo los textos y los discursos evolucionan y cambian en diferentes contextos, el DHA identificó cuatro capas para describirlos (Reisigl \& Wodak, 2016). La primera capa se identifica con las relaciones inmediatas entre 
expresiones, co-textos y co-discursos. La segunda capa describe las relaciones intertextuales e interdiscursivas, por ejemplo, cómo se relacionan todos los textos con otros textos (intertextualidad) o cómo los discursos orientados a temas se vinculan con otros en diferentes formas como relaciones interdiscursivas, como en este caso, la práctica de la dual screening. Una tercera capa se relaciona con las variables externas como variables extra-lingüísticas o sociológicas, un contexto de situación, que en este caso puede relacionarse con la ecología de los medios en la esfera política, y finalmente, la cuarta se considera la histórica y sociopolítica. El contexto y los textos relacionados, que en este caso están vinculados al contexto histórico y político de Chile.

El análisis discursivo desde el enfoque histórico-discursivo identifica cinco estrategias discursivas (Reisigl \& Wodak, 2016). Reisigl y Wodak (2016) describen una estrategia como un plan de acción para lograr un objetivo específico. Estas estrategias discursivas son la nominación, predicación, argumentación, perspectivación y mitigación.

Tabla 2. Estrategias discursivas identificadas por el enfoque histórico-discursivo (Reisigl \& Wodak, 2016)

\begin{tabular}{|l|l|}
\hline Estrategia & Descripción \\
\hline Nominación & $\begin{array}{l}\text { Enfocada en la construcción discursiva de actores sociales, objetos, } \\
\text { eventos, procesos y acciones. Puede expresarse en diferentes } \\
\text { dispositivos, como categorización de actores sociales, deixis, tropos, } \\
\text { verbos y sustantivos para denotar procesos, entre otros. }\end{array}$ \\
\hline Predicación & $\begin{array}{l}\text { Centrada en cómo se representan los actores sociales en el discurso } \\
\text { (negativa o positivamente). Esta estrategia se puede definir como la } \\
\text { calificación discursiva de actores, objetos, eventos, procesos y acciones } \\
\text { sociales. Esta categoría incluye atribuciones evaluativas estereotipadas, } \\
\text { sustantivos predicados o predicados explícitamente, colocaciones, } \\
\text { figuras retóricas, entre otros. }\end{array}$ \\
\hline Argumentación & $\begin{array}{l}\text { Cuestionamientos o justificaciones de verdad y derecho. Incluye topoi } \\
\text { y falacias. }\end{array}$ \\
\hline Perspectivación & $\begin{array}{l}\text { expresa la posición del hablante y su implicación o distancia, y punto } \\
\text { de vista. Por ejemplo, el uso de deixis, discurso directo / indirecto, } \\
\text { metáforas, prosodia animada, etc. }\end{array}$ \\
\hline Intensificación/ mitigación & $\begin{array}{l}\text { Modificación de la fuerza ilocucionaria y el estado epistémico de una } \\
\text { proposición. Puede identificarse con diminutivos / aumentativos, } \\
\text { partículas modales, hipérboles, actos de habla indirectos o verbos de } \\
\text { decir, sentir y pensar, entre otros. }\end{array}$ \\
\hline
\end{tabular}

Estas estrategias discursivas analizan diversos aspectos delos textos y ayudan a comprender cómo se construyen los discursos en este contexto comunicativo. Sin embargo, esta investigación se ha centrado en las estrategias argumentativas, específicamente en los topoi y falacias presentes en los textos de Twitter marcados con los hashtags de cinco programas políticos chilenos.

Además, analizar los argumentos y las estrategias argumentativas puede ser útil para comprender cómo se realizan los debates en las redes sociales, en términos de lo que se considera veraz o correcto en estos contextos y cómo los usuarios justifican y respaldan sus afirmaciones. Este tipo de análisis puede ayudar a identificar qué atribuciones, ni las positivas ni las negativas, están justificadas a través de topoi. Los topoi son "partes de la argumentación que pertenecen a las premisas obligatorias de un argumento, ya sea explícito o tácito" (Wodak, 2015, p. 35). Como explican Reisigl y Wodak (2016), los topoi son garantías que conectan los argumentos con sus afirmaciones o conclusiones centrales y son socialmente convencionalizados, por esa razón es importante analizar los argumentos falaces que se utilizan ampliamente en la discusión política Reisigl (2014) afirma que los topoi son conclusiones relacionadas con el contenido que son recurrentes en un determinado campo social, disciplina o teoría. Los topos se utilizan como 
una estrategia de argumentación para justificar atribuciones positivas o negativas y pueden considerarse razonables, pero también falaces. La siguiente tabla muestra algunos de los topoi presentes en la literatura con ejemplos extraídos del conjunto de datos de esta investigación (Wodak, 2001; Wodak 2011; Reisigl, 2014):

Tabla 3. Ejemplos de análisis de estrategias argumentativas

\begin{tabular}{|c|c|c|}
\hline TOPOS & CONCLUSIÓN/GARANTÍA & EJEMPLOS DE LOS DATOS \\
\hline Topos de la carga & $\begin{array}{l}\text { Si una institución está agobiada por un } \\
\text { problema específico, entonces uno debe } \\
\text { actuar para disminuir }\end{array}$ & $\begin{array}{l}\text { \#ElInformante Para financiar la } \\
\text { gratuidad, hay que ordenar la casa. } \\
\text { Reforma Tributaria, Penal, Laboral, } \\
\text { Fomento a la Investigación... etc... }\end{array}$ \\
\hline Topos de urgencia & $\begin{array}{l}\text { Las decisiones / acciones deben llevarse } \\
\text { a cabo muy rápidamente debido a } \\
\text { eventos importantes que van más allá } \\
\text { de las responsabilidades del usuario. }\end{array}$ & $\begin{array}{l}\text { 1. El Sistema de Pensiones en Chile esta } \\
\text { a punto de colapsar y pronto generará } \\
\text { un problema social y de pobreza grave } \\
\text { \#elinformante }\end{array}$ \\
\hline Topos de autoridad & $\begin{array}{l}\text { Si alguien con autoridad aprobó } \\
\text { una determinada acción / dice algo, } \\
\text { entonces la acción debe llevarse a cabo } \\
\text { / debe ser verdad. }\end{array}$ & $\begin{array}{l}\text { No se trata de ideologizar de debate. } \\
\text { Es un tema de DDHH reproductivos } \\
\text { básicos apoyado por la ONU \# enacional }\end{array}$ \\
\hline $\begin{array}{l}\text { Topos historia est } \\
\text { magistra vitae }\end{array}$ & $\begin{array}{l}\text { Si la historia demuestra que una } \\
\text { acción o decisión específica tiene } \\
\text { ciertas consecuencias favorables o } \\
\text { desfavorables, se debe realizar u omitir } \\
\text { esa acción o decisión específica }\end{array}$ & $\begin{array}{l}\text { \#enacional Mal Saffirio el inicio de la “ } \\
\text { cuestión Palestina” también ocurrió en } \\
\text { el siglo pasado, entonces olvidemos las } \\
\text { masacres. }\end{array}$ \\
\hline Topos de justicia & $\begin{array}{l}\text { Si algo o alguien son iguales en ciertos } \\
\text { aspectos, deben ser tratados de la } \\
\text { misma manera. Igualdad ante la ley }\end{array}$ & $\begin{array}{l}\text { \#ENacional mal si las leyes son para } \\
\text { defender a los mismos de siempre, } \\
\text { olvidando que todos debemos ser } \\
\text { iguales ante la ley. }\end{array}$ \\
\hline $\begin{array}{l}\text { Topos de los } \\
\text { derechos humanos }\end{array}$ & $\begin{array}{l}\text { Si una acción o decisión no sigue las } \\
\text { convenciones de derechos humanos, no } \\
\text { debe llevarse a cabo }\end{array}$ & $\begin{array}{l}\text { Donde están los paladines de los ddhh } \\
\text { frente a esta grave violación de los } \\
\text { derechos humanos de NIÑOS }\end{array}$ \\
\hline $\begin{array}{lr}\text { Topos de la } \\
\text { la decencia } \\
\text { resposabilidad }\end{array}$ & $\begin{array}{l}\text { Si alguien no es decente / respetable, no } \\
\text { debe ser un político. }\end{array}$ & $\begin{array}{l}\text { Javiera Blanco está metiendo } \\
\text { demasiado ruido, deberia dar un paso al } \\
\text { costado silenciosamente... }\end{array}$ \\
\hline
\end{tabular}

La tabla anterior presenta diversos textos extraídos del corpus de análisis que sirven para ejemplificar las diversas estrategias argumentativas presentes en tweets relacionados con programas de televisión políticos. Estos textos muestran parte del debate político llevado acabo en este tipo de contextos y como los topoi sirven para analizar la comunicación política y cómo se articula la discusión en torno a diversos participantes de la esfera pública.

\section{Hacia un análisis de los componentes multimodales en la argumentación}

Herring (2013) plantea que los textos de redes sociales poseen diversas características multimodales, tales como diversos tipos de imágenes, animaciones, entre otros. Para comprender el lenguaje online, es necesario entender cómo los diferentes modos funcionan juntos para formar textos en línea coherentes y significativos. Los modos, que también se conocen como modos comunicativos o modos semióticos, se refieren en términos generales a sistemas o recursos que las personas utilizan para crear significado. Estos incluyen el lenguaje hablado, el lenguaje escrito, la imagen, el sonido, el gesto, etc.

Una de las principales características de los textos digitales es su naturaleza multimodal (KhosraviNik, 2017), por lo tanto, el análisis de los recursos argumentativos en este tipo de 
textos debiese considerar los diversos modos para la caracterización del debate en contextos híbridos. Como plantean Richardson y Wodak (2009), los elementos visuales pueden constituir argumentos, en el sentido que pueden justificar positiva o negativamente diversos puntos de vista, como presentan los siguientes ejemplos:

Figura 6. Ejemplo de análisis de elementos multimodales

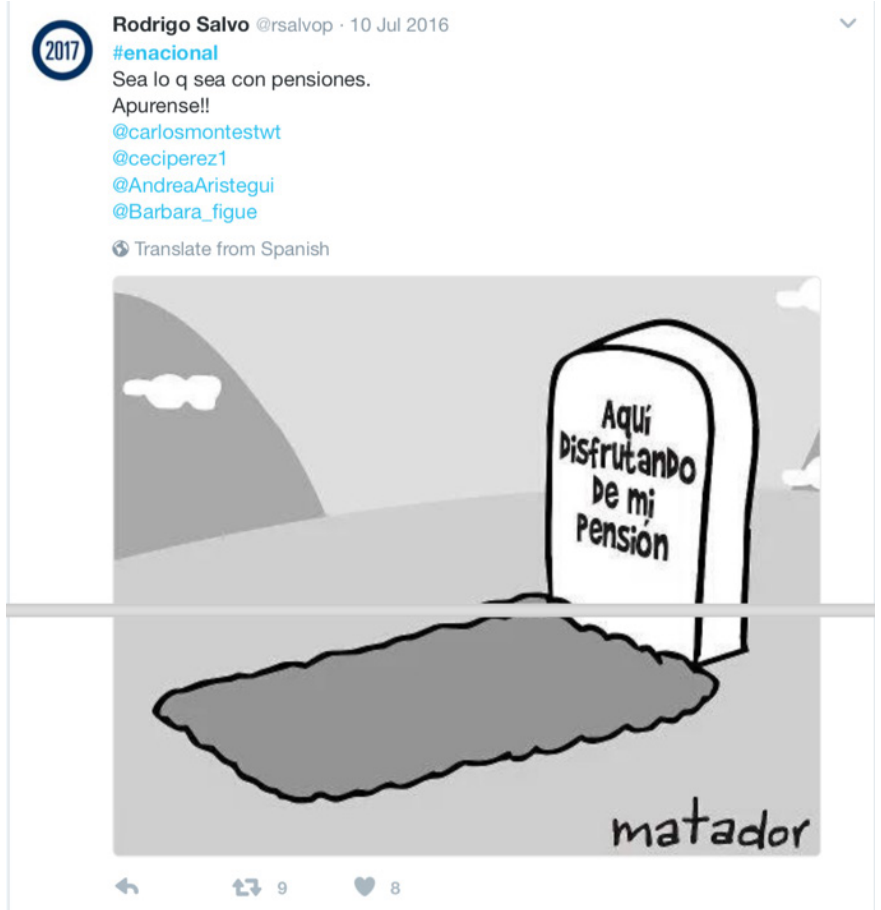

La figura 6 muestra un tweet relacionado con la crisis del sistema de capitalización individual como modelo de seguridad social, como lo son las AFPs. En este caso, el tweet no especifica un plan o acción determinados para abordar el sistema de pensiones actualmente vigente, sino que pide a los políticos que hagan algo para cambiar esta situación rápidamente, lo cual se puede clasificar como topos de la urgencia. Este topos tiene como premisa en actuar con urgencia para evitar consecuencias desfavorables para ciertos grupos. Para hacer esto, el usuario incluye los nombres de usuario de los políticos de diferentes orientaciones políticas que son invitados en el programa de televisión en la discusión sobre el sistema de pensiones, dirigiéndose directamente a ellos (@carlosmontestwt, @ ceciperez1, @AndreaAristegui y @Barbara_figue).El tweet también declara explícitamente la urgencia del asunto pidiendo a los destinatarios que se den prisa. Esto debido a que el sistema pensiones está en crisis, las personas envejecen y se jubilan con pensiones muy pobres que no son suficientes para satisfacer las necesidades básicas. Dentro de los recursos utilizados en el texto, el usuario integra una caricatura para ilustrar las consecuencias de la falta de acción de parte de la clase política. Como afirma Van Leeuwen (2009), las representaciones de las caricaturas pueden considerarse metáforas visuales, en este caso la tumba representaría la ineficiencia del sistema. La caricatura muestra una tumba con la inscripción "aquí disfrutando mi pensión" lo cual tiene directa relación con la discusión de la extensión de la vida laboral, propuesta como solución para el mejoramiento de las pensiones.

Otro ejemplo de texto con diversos elementos multimodales es la figura 7, la cual presenta un topos de la responsabilidad o decencia, que plantea que si alguien no es decente y respetable, no debe ser político. 
Figura 7. Ejemplo de análisis de elementos multimodales

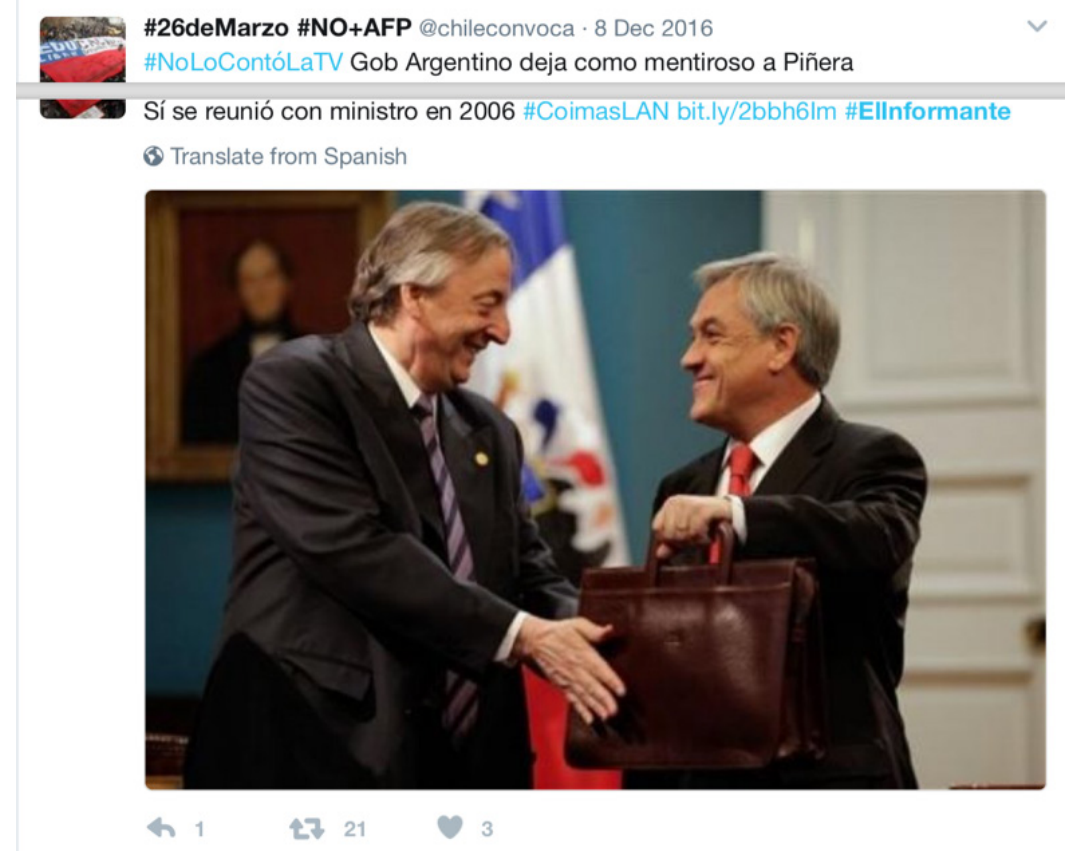

Este topos de decencia muestra un tweet relacionado con Sebastian Piñera y su participación en casos de corrupción en Argentina, específicamente, el uso de sobornos a políticos para autorizar su aerolínea (LAN). Esto tiene la intención de implicar que no es apto para la política. Esta declaración se hace en el contexto del anuncio de que tenía la intención de postularse para la reelección en el programa de televisión El Informante (8-12-2016). Para respaldar la afirmación de que Piñera estuvo involucrado en casos de corrupción, el tweet incluye un hipervínculo a un artículo que demuestra su participación en pagos ilegales. El usuario declara que la información proporcionada por el enlace no está disponible en las emisoras de televisión chilenas con la inclusión del hashtag "\#NoLoContóLaTV". Además, el usuario incluye otro hashtag "\#CoimasLAN" que vincula este tweet con la conversación sobre sobornos relacionados con la aerolínea propiedad del ex presidente. Otro tipo de evidencia presentada en el tweet para respaldar esa afirmación es una imagen de Sebastián Piñera y Néstor Kirchner (ex presidente de Argentina) intercambiando un maletín.

En este caso, la imagen puede ser engañosa, porque la imagen no está relacionada con el caso de corrupción y muestra una visita oficial del ex presidente de Argentina a La Moneda, constituyéndose como un elemento falaz. La imagen muestra una conexión entre los políticos pero no prueba ninguna ilegalidad o actividad sospechosa. Esto puede considerarse como una falacia del hombre de paja en la que el usuario tergiversa a la otra parte en su tweet, atribuyéndole un punto de vista o comportamiento ficticio. En el ejemplo, el punto de vista ficticio podría ser que la imagen junto con los tweets insinúa que los sobornos están realmente en la maleta o que la imagen muestra un intercambio ilegal. El texto en el tuit sugiere que Piñera estuvo involucrado en actividades ilegales. Sin embargo, la imagen muestra una actividad oficial, que se recontextualiza y presenta como parte del caso de corrupción descrito anteriormente. El topos de la decencia o la respetabilidad se utiliza en estos casos para señalar el comportamiento inapropiado de las figuras públicas, estableciendo estándares éticos para las personas que están a cargo de la toma de decisiones que afecta a la población.

Este tipo de análsis argumentativo que considera un aspecto multimodal se erige como una 
primera aproximación a la argumentación en medios digitales relacionados a medios televisivos. La inclusión de aspectos multimodales puede ser util para la exploración de cómo se constituyen los argumentos en este tipo de discusión online y qué tipos de recursos utilizan los usuarios de las redes para interactuar en relación a estos temas.

\section{Discusión y aportes de la investigación}

Este tipo de estudio contribuye a los estudios críticos del discurso en la exploración de un fenómeno relativamente nuevo, que puede ser la comunicación digital en el sistema híbrido de medios. Como se mencionó, la investigación de la comunicación política en el sistema híbrido de medios supone un nuevo desafío y un área prometedora para los estudios del discurso, que permiten explorar cómo se reconfiguran los medios y las estructuras de poder en este nuevo contexto de medios (Gruber, 2017).

Además, investigaciones adicionales podrían considerar otros contextos sociopolíticos de producción en los cuales este tipo de estudio podría ser replicado, analizando estrategias argumentativas en contextos de dual screening. Esto podría ayudar a describir con más detalle la esfera pública y su relación en el sistema de medios híbridos en contextos latinoamericanos, proporcionando nueva información sobre las relaciones políticas y las prácticas digitales en el área.

El análisis de las estrategias argumentativas en este tipo de práctica social contribuye a la exploración discursiva del fenómeno del dual-screening y en consecuencia al estudio del sistema híbrido de medios. El aspecto multimodal estudiado en esta muestra de textos se erige como una primera aproximación al análisis de aspectos argumentativos multimodales, tanto falacias como topoi y sus aspectos visuales, que se puede desarrollar en futuras investigaciones.

La investigación sobre el sistema híbrido de medios no está restringida a la relación entre la televisión y las redes sociales. Este tipo de estudio en el sistema de medios híbridos puede integrar múltiples lógicas de medios y diferentes plataformas, como medios de radio o impresos. Las investigaciones futuras pueden explorar las diferentes intersecciones entre los medios y explorar diversas plataformas de redes sociales.

Otra posibilidad para la investigación es la exploración de otros tipos de discusión política en los medios de difusión. Un ejemplo de esto puede ser el estudio de otros formatos y géneros de televisión, como programas matinales, noticias o programas de trasnoche. El estudio de otro tipo de programas televisados puede ayudar a explorar otras manifestaciones de la política cotidiana en línea. A pesar de que estos géneros diferentes no son exclusivamente sobre política, incluyen un enfoque diferente del debate sobre cuestiones políticas y participación ciudadana, que puede contribuir al estudio adicional de la esfera pública y la deliberación política en diversos contextos.

\section{Referencias bibliográficas}

Altheide, D. (2013). Media Logic, Social Control, and Fear. Communication Theory, 23(3), 223238.

Baker, P. (2006). Using Corpora in Discourse Analysis. London: Continuum

Baker, P., Gabrielatos, C., KhosraviNik, M., Krzyżanowski, M., McEnery, T., \& Wodak, R. (2008). A useful methodological synergy? Combining critical discourse analysis and corpus linguistics to examine discourses of refugees and asylum seekers in the UK press. Discourse \& Society, 19(3), 273- 306. https://doi.org/10.1177/0957926508088962

Barton, D., \& Lee, C. (2013). Language online. London: Routledge 
Boyd, d., \& Crawford, K. (2012). Critical questions for big data: Provocations for a cultural, technological, and scholarly phenomenon. Information, Communication \& Society, 15(5), 662679.

Cameron, J., \& Geidner, N. (2014). Something Old, Something New, Something Borrowed from Something Blue: Experiments on Dual Viewing TV and Twitter. Journal of Broadcasting \& Electronic Media, 58(3), 400-419.

Chadwick, A. (2013). The hybrid media system: Politics and power. New York, NY: Oxford University Press

Flick, U. (2008). Designing Qualitative Research. London: SAGE Publications Ltd.

Gil de Zúñiga, H., Garcia-Perdomo, V., \& McGregor, S. C. (2015). What Is Second Screening? Exploring Motivations of Second Screen Use and Its Effect on Online Political Participation. Journal of Communication, 65(5), 793-815.

Gruber, H. (2017). Genres of political communication in Web 2.0. En R. Wodak \& B. Forchtner (Eds.), The Routledge Handbook of Language and Politics (pp. 412-425). Routledge.

Hardaker, C., \& McGlashan, M. (2016). "Real men don't hate women”: Twitter rape threats and group identity. Journal of Pragmatics, 91, 80-93.

Herring, S. C. (2013). Discourse in Web 2.0: Familiar, reconfigured, and emergent. En D. Tannen \& A.M. Tester (Eds.), Discourse 2.0: Language and new media (pp. 1-25). Washington DC: Georgetown University Press.

KhosraviNik, M. (2017). Social Media Critical Discourse Studies (SM-CDS). En J. Flowerdew \& J. Richardson (Eds.), Handbook of Critical Discourse Studies (pp. 582-596). London: Routledge.

KhosraviNik, M., \& Unger, J. W. (2016). Critical discourse studies and social media: Power, resistance and critique in changing media ecologies. En R. Wodak \& M. Meyer (Eds.), Methods of critical discourse studies (pp. 63-94). London: SAGE

Latzko-Toth, G., Bonneau, C. \& Millette, M. (2017). Small data, thick data: Thickening strategies for trace-based social media research. En L. Sloan \& A. Quan-Haase (Eds.), The SAGE Handbook of Social Media Research Methods (pp. 199-214). Thousand Oaks: SAGE.

Lemke, J. (2009). Multimodal genres and transmedia traversals: Social semiotics and the political economy of the sign. Semiotica, 173, 283-297.

Marchetti, R., \& Ceccobelli, D. (2015). Twitter and Television in a Hybrid Media System. Journalism Practice, 2786(October), 1-19.

Marwick, A. (2014). Ethnographic and Qualitative Research on Twitter. En K. Weller, A. Bruns, C. Puschmann, J. Burgess \& M. Mahrt (Eds), Twitter and Society (109-122). New York: Peter Lang.

Page, R. (2012). The linguistics of self-branding and micro-celebrity in Twitter: The role of hashtags. Discourse \& Communication, 6(2), 181-201.

Page, R., Barton, D., Unger, J., \& Zappavigna, M. (2014). Researching language and social media: a student guide. London: Routledge

Potts, A., Simm, W., Whittle, J., \& Unger, J. W. (2014). Exploring 'success' in digitally augmented activism: A triangulated approach to analyzing UK activist Twitter use. Discourse, Context and 
Media, 6, 65-76. https://doi.org/10.1016/j.dcm.2014.08.008

Reisigl, M. (2014). Argumentation analysis and the Discourse-Historical Approach. A methodological framework. En C. Hart \& P. Cap (Eds.), Contemporary Critical (pp. 67-96). London: Bloomsbury Academic. doi.org/10.5040/9781472593634.ch-003

Reisigl, M. \& Wodak, R. (2016). The discourse-historical approach (DHA). En R. Wodak \& M. Meyer (Eds.) Methods of critical discourse studies (pp. 23-61). London: Sage.

Richardson, J.E., \& Wodak, R. (2009). The impact of visual racism: Visual arguments in political leaflets of Austrian and British Far-right Parties. Controversia, 6 (2). 45-77.

Roberts, C. (2008). A Discourse Analysis Approach to the Social Functions of Humour, with Reference to the Political Panel Discussion Programme, Question Time (Tesis doctoral), Lancaster University, Lancaster, Reino Unido.

Saldaña, J. (2013). The coding manual for qualitative researchers. London: SAGE.

Silverman, D. (2016). Qualitative research. London: SAGE.

Vaccari, C., Chadwick, A. \& O'Loughlin, B. (2015). Dual Screening the Political: Media Events, Social Media, and Citizen Engagement. Journal of Communication, 65(6), 1041-1061.

Van Leeuwen, T. (2008). Discourse and Practice: New tools for critical discourse analysis. Oxford: Oxford University Press. https://doi.org/10.1093/acprof

Van Leeuwen, T. (2009). Discourse as the recontextualization of social practice: A guide. En R. Wodak \& M. Meyer (Eds.), Methods of critical discourse analysis (2da edición) (pp. 144-161). London: SAGE

Unger, J.W., Wodak, R. \& KhosraviNik, M. (2016). Critical discourse studies and social media data. En D. Silverman (Ed.), Qualitative research (pp. 277-293). London: Sage.

Wodak, R. (2001). The discourse-historical approach. En R. Wodak \& M. Meyer (Eds.), Methods of critical discourse analysis (pp. 63-94). London: SAGE

Wodak, R. (2011). The discourse of politics in action: Politics as usual. Basingstoke: Palgrave Macmillan.

Wodak, R. (2015). The politics of fear: What right-wing populist discourses mean. London: Sage.

Zappavigna, M. (2012). Discourse of Twitter and Social Media: How we use Language to Create Affiliation on the Web. London: Continuum

Zappavigna, M. (2017) Ambient liveness: Searchable audiences and second screens. En C. Hight \& H. Harindranath (Eds.), Studying Digital Media Audiences: Perspectives from Australasia (pp. 150-172). London: Routledge 